\title{
Correction to: MYEOV increases HES1 expression and promotes pancreatic cancer progression by enhancing SOX9 transactivity
}

\author{
Erbo Liang $\cdot$ Yishi Lu $(\mathbb{D}) \cdot$ Yanqiang Shi $\cdot$ Qian Zhou $\cdot$ Fachao Zhi $\mathbb{D}$
}

Published online: 3 February 2021

(c) The Author(s), under exclusive licence to Springer Nature Limited 2021

Correction to: Oncogene (2020) 39:6437-6450

https://doi.org/10.1038/s41388-020-01443-4

published online 02 September 2020

Unfortunately, the name of the university and the department were given in three different representations, although they are the same affiliation.
Below is the correct affiliation for all authors:

Guangdong Provincial Key Laboratory of Gastroenterology, Institute of Gastroenterology of Guangdong Province, Department of Gastroenterology, Nanfang Hospital, Southern Medical University, 510515 Guangzhou, China The original article has been corrected. 